\title{
HESS J1641-463, a very hard spectrum TeV gamma-ray source in the Galactic plane.
}

\author{
I. Oya* \\ DESY, Zeuthen, Germany \\ E-mail: igor.oya.vallejo@desy.de

\section{S. Casanova} \\ Instytut Fizyki Jądrowej PAN, Kraków, Poland \\ Max-Planck-Institut für Kernphysik, Heidelberg, Germany \\ E-mail: sabrina.casanova@ifj.edu.pl, sabrina.casanova@mpi-hd.mpg.de
}

\section{F. Aharonian}

Max-Planck-Institut für Kernphysik, Heidelberg, Germany

Dublin Institute for Advanced Studies, Dublin 2, Ireland

\section{Dalton}

Université Bordeaux 1, CNRS/IN2P3, Gradignan, France

Now at: Active Space Technologies GmbH, Berlin, Germany

\section{For the H.E.S.S. collaboration}

HESS J1641-463 is a unique source discovered by the High Energy Stereoscopic System (H.E.S.S.) telescope array in the multi-TeV domain. The source had been previously hidden in the extended tail of emission from the bright nearby source HESS J1640-465. However, the analysis of the very-high-energy (VHE) data from the region at energies above $4 \mathrm{TeV}$ revealed this new source at a significance level of $8.5 \sigma$. HESS J1641-463 showed a moderate flux level $\mathrm{F}(\mathrm{E}>1 \mathrm{TeV})=\left(3.64 \pm 0.44_{\text {stat }} \pm 0.73_{\text {sys }}\right) \times 10^{-13} \mathrm{~cm}^{-2} \mathrm{~s}^{-1}$, corresponding to $1.8 \%$ of the Crab Nebula flux above the same energy, and a hard spectrum with a photon index $\Gamma=2.07 \pm 0.11_{\text {stat }}$ $\pm 0.20_{\text {sys }}$. The light curve was investigated for evidence of variability, but none was found on both short (28-min observation) and long (yearly) timescales. HESS J1641-463 is positionally coincident with the radio supernova remnant (SNR) G338.5+0.1. There is no clear X-ray counterpart of the SNR, although Chandra and XMM-Newton data reveal some weak emission that may be associated. If the emission from HESS J1641-463 is produced by cosmic ray protons colliding with the ambient gas, then the proton spectrum extends up to $0.1 \mathrm{PeV}$ (99\% confidence level) and likely to higher energies, $>0.27 \mathrm{PeV}$ (90\% confidence level). If this is the case, then HESS J1641-463 may be a member of a larger source population contributing to the Galactic cosmic-ray flux around the knee.

The 34th International Cosmic Ray Conference,

30 July- 6 August, 2015

The Hague, The Netherlands

\footnotetext{
${ }^{*}$ Speaker.
} 


\section{H.E.S.S. observations and analysis}

The data used for the analysis described here were taken with High Energy Stereoscopic System (H.E.S.S.) between 2004 and 2011, amounting to an acceptance-corrected livetime of 72 hours. The events were reconstructed using a Hillas parameter technique [1]. The results where crosschecked using independent methods [2,3], yielding consistent results.

HESS J1641 - 463(J1641 -463 hereafter) was not visible in the original HESS J1640-465 images, without energy cut in the events, due to the much brighter, nearby source HESS J1640-465. However, thanks to the improved H.E.S.S. point spread function (PSF) at higher energies, and to its hard spectrum, J1641-463 was clearly visible when increasing the energy threshold, where the contamination from HESS J1640-465 was reduced. The VHE $\gamma$-ray excess image obtained for $\mathrm{E}>4 \mathrm{TeV}$ is shown in Fig. 1, left, where the background level is estimated following the ring background model [4]. Figure 1, right, shows the projection of the excess events in the rectangular region shown in Fig. 1, left, for different energy bands.

We fitted the differential VHE $\gamma$-ray spectrum of $\mathrm{J} 1641-463$ with a power-law function $\mathrm{d} N / \mathrm{d} E=$ $\phi_{0} \times(E / 1 \mathrm{TeV})^{-\Gamma}$ in the range from $0.64 \mathrm{TeV}$ to $100 \mathrm{TeV}$ using the forward-folding technique [5], the best fitting parameters being $\phi_{0}=\left(3.91 \pm 0.69_{\text {stat }} \pm 0.78_{\text {sys }}\right) \times 10^{-13} \mathrm{~cm}^{-2} \mathrm{~s}^{-1} \mathrm{TeV}^{-1}$ and $\Gamma=$ $2.07 \pm 0.11_{\text {stat }} \pm 0.20_{\text {sys }}$. The flux level is $\phi(\mathrm{E}>1 \mathrm{TeV})=\left(3.64 \pm 0.44_{\text {stat }} \pm 0.73_{\text {sys }}\right) \times 10^{-13} \mathrm{~cm}^{-2} \mathrm{~s}^{-1}$, corresponding to $1.8 \%$ of the Crab Nebula flux above the same energy. The light curve was investigated for evidence of variability, but none was found on both short (28-min observation) and long (yearly) timescales.

\section{Search for multiwavelength counterpart}

\subsection{In the radio band}

J1641-463 is found within the bounds of the supernova remnant (SNR) G338.5+0.1 [6]. No precise estimates on the age of this SNR exist. Depending on its physical size, however, its age could range between 1.1 to $1.7 \mathrm{kyr}$ [7].

The distribution of molecular gas around J1641-463 is shown in the top left inset of Fig. 1. This distribution is obtained by integrating the $\mathrm{CO} 1 \rightarrow 0$ rotational line emission over a range in velocity between $-40 \mathrm{~km} / \mathrm{s}$ to $-30 \mathrm{~km} / \mathrm{s}[8,9]$. The choice of this range is motivated by the presence of dense molecular cloud (MC) clumps in the region [10]. Using the model for the Galactic rotation curves by Kothes \& Dougherty [11] the gas is located at a distance of $\sim 11 \mathrm{kpc}$. Assuming a ratio $X_{\mathrm{CO}->N_{\mathrm{H}_{2}}}=1.5 \times 10^{20}$ between the $\mathrm{CO}$ velocity integrated intensity and the column density of molecular gas, $N_{H_{2}}$, the total column density from the extraction region of J1641-463 is $1.7 \times 10^{22}$ $\mathrm{cm}^{-2}$. At $11 \mathrm{kpc}$ the density and the total mass of the gas clumps inside the source region are about $100 \mathrm{~cm}^{-3}$ and $2.4 \times 10^{5}$ solar masses, respectively.

\subsection{In the X-rays}

No candidate for an X-ray counterpart of J1641-463 was found in existing catalogs. Two data sets from Chandra (ObsID 11008 and ObsID 12508) and one from XMM-Newton (ObsID 

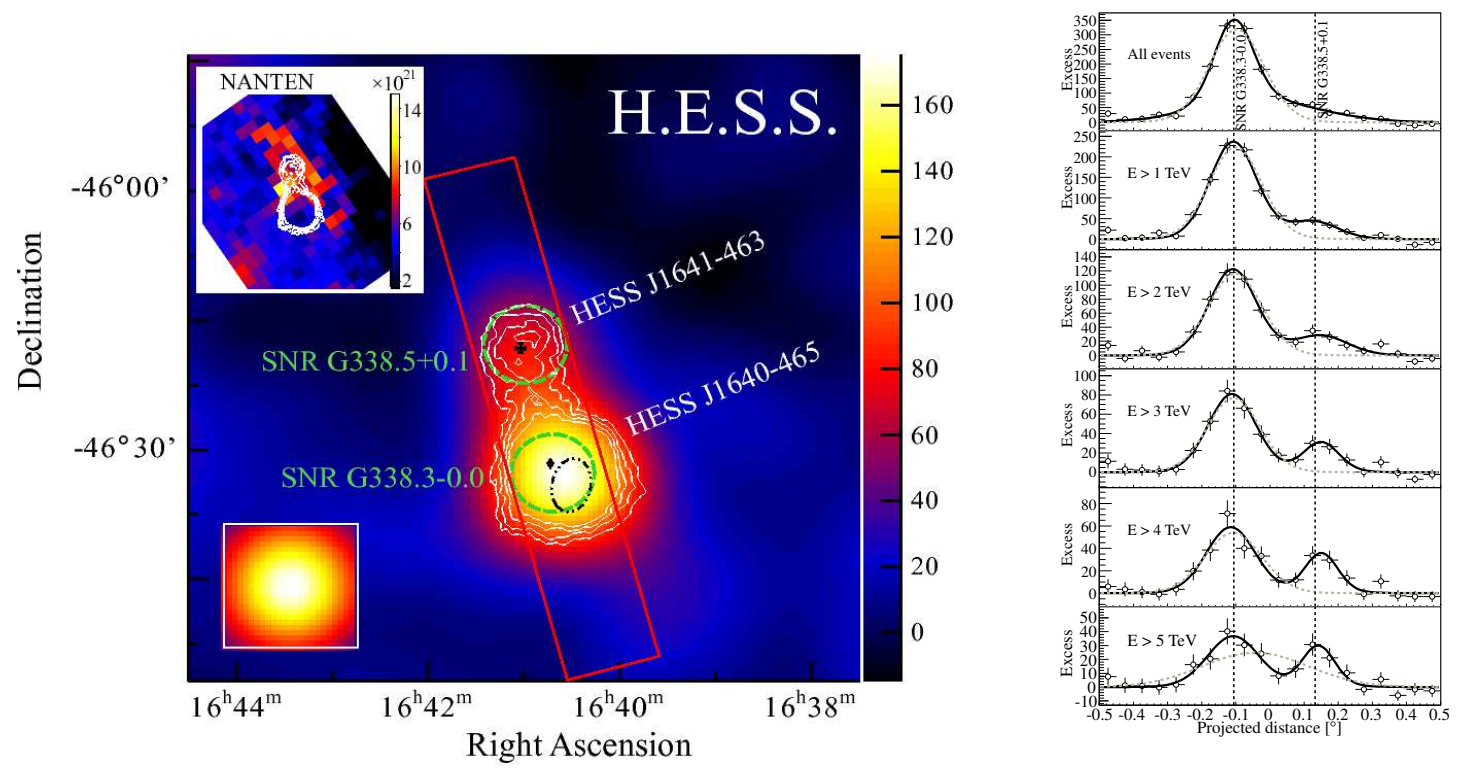

Figure 1: Left: Map of excess events with energies $\mathrm{E}>4 \mathrm{TeV}$ for the region around $\mathrm{J} 1641-463$ smoothed with the instrument PSF (shown inside the white box). The white contours indicate the significance of the emission at the 5, 6, 7 and $8 \sigma$ level. The black cross indicates the best fit position of the source, the green dashed circles show the positions and approximate extensions of the two nearby SNRs, the black diamond the position of PSR J1640-4631, the dash-dotted black ellipse the 95\% confidence error position of 1FHL J1640.5-4634, and the red box indicates the area for the extraction of the profiles shown in the right panel. The upper left inset shows a map of the distribution of the column density of molecular hydrogen in units of $\mathrm{cm}^{-2}$, estimated from the NANTEN $\mathrm{CO}(1-0)$ data, together with the H.E.S.S. significance contours. Right: Distribution of VHE $\gamma$-ray excess profiles and Gaussian fits for the red rectangular slice shown in the left panel. Vertical lines show the position of the SNR 338.3-0.0 and G338.5+0.1. Figures from [7].

0302560201) were inspected in order to search for an X-ray counterpart of J1641-463. The outcome of this analysis, described in detail in ref. [7], is that no obvious counterpart of J1641-463 could be established neither in the Chandra nor the XMM-Newton data.

\subsection{In the high energy $\gamma$-rays}

Lemoine-Goumard et al. [12] report the detection of two distinct sources with Fermi-LAT above $100 \mathrm{MeV}$, corresponding to the positions of J1641-463 and HESS J1640-465. The softest emission in this region as seen by Fermi-LAT corresponds to J1641-463, which is well fitted with a power law of index $\Gamma=2.47 \pm 0.05 \pm 0.06$ and presents no significant gamma-ray signal above $10 \mathrm{GeV}$. The connection between this steep spectrum and the hard H.E.S.S. spectrum remains unclear, although the Fermi-LAT and H.E.S.S results suggest two different mechanisms, or sources, producing the $\gamma$-ray emission.

\section{Scenarios to explain the $\mathbf{J 1 6 4 1}-463$ emission}

A first possible scenario to explain the J1641-463 emission is that the SNR G338.5+0.1 is young and is able to accelerate particles up to hundreds of TeV. The left panel of Fig. 2 shows 


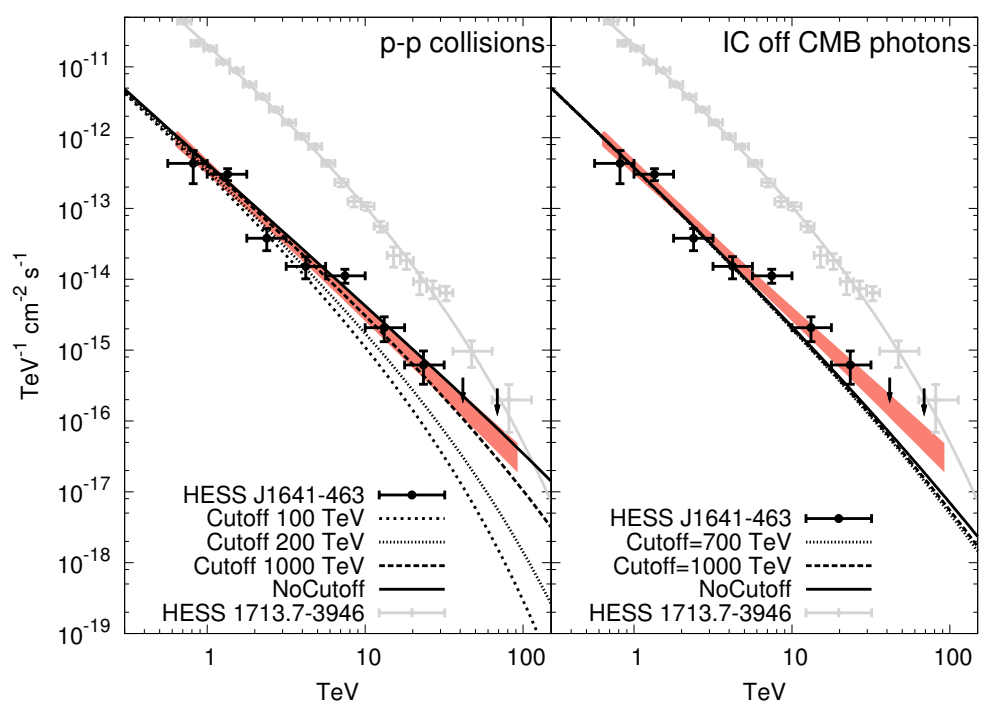

Figure 2: Differential $\gamma$-ray spectrum of J1641-463 together with the expected emission from p-p collisions (left) and IC off CMB photons (right). The pink area represents the $1 \sigma$ confidence region for the fit to a power law model, the black data points the H.E.S.S. measured photon flux, the arrows the 95\% CL upper limits on the flux level, and the black curves the expected emission from the models. For comparison, the gray data points and curve represent the archival data of SNR RX J1713.7-3946 [13]. Figure from [7].

the comparison between the H.E.S.S. spectrum and the spectrum produced by accelerated protons from G338.5+0.1, interacting with the ambient gas. The predicted spectra are calculated using the parametrization of Kelner et al. [14], assuming a proton spectrum with a power-law slope of -2.1 . The 99\% confidence level (CL) lower limit on the proton cutoff energy corresponds to $100 \mathrm{TeV}$. Remarkably, the $\gamma$-ray spectrum of J1641-463 is harder than that observed from the young SNR RXJ1713-4936 at energies above few TeV [13]. With a proton spectrum extending almost up to 1 $\mathrm{PeV}, \mathrm{J} 1641-463$ may be a representative of a new source type that is contributing significantly to the galactic cosmic-rays flux around the knee.

If G338.5+0.1 is older, VHE protons accelerated by the young SNR G338.3-0.0, positionally coincident with HESS J1640-465 [15], could have already reached the dense MC coincident with J1641-463. This would explain the relatively high brightness of J1641-463 in comparison with HESS J1640-465 at high energies. In such a scenario, the much younger adjacent G338.3-0.0 would be a major source of CRs.

Electrons of hundreds of $\mathrm{TeV}$ inverse Compton (IC) scattering off the cosmic microwave background photons (CMB) could explain the emission from J1641-463. These electrons would be accelerated either in G338.5+0.1 or in the PWN associated to the young energetic pulsar, PSR J1640-4631 [16]. Even assuming a pure power law for the primary electron spectrum, the cross section for IC scattering decreases at high energies resulting in a break in the $\gamma$-ray spectrum at multi $\mathrm{TeV}$ energies, not observed in the spectrum of J1641-463. The predicted IC radiation, shown in the right panel of Fig. 2, was obtained by assuming that the electron cooled spectrum is a power law of spectral index -3.14 . The $99 \%$ CL lower limit on the cutoff energy corresponds to $700 \mathrm{TeV}$. We note that it is extremely difficult to accelerate electrons in SNRs to such energies as hundred $\mathrm{TeV}$ electrons suffer severe synchrotron losses in the amplified magnetic fields of the 
acceleration sites.

\section{Conclusions}

We present a new unique VHE source, showing one of the hardest $\gamma$-ray spectra ever found at these energies. In order to explain the observed VHE $\gamma$-ray spectrum, scenarios where protons are accelerated up to hundreds of $\mathrm{TeV}$ at either G338.5+0.1 or G338.3-0.0, and then interact with local gas or nearby massive MCs are the most compelling ones. In such scenario, J1641-463 would be a representative of a source class contributing to the Galactic cosmic-ray flux around the knee. Otherwise, a leptonic emission-based scenario cannot be formally ruled out, although it is severely constrained by the absence of breaks in the $\gamma$-ray spectrum and by the high efficiency required for an accelerator located within the SNR, where strong losses through synchrotron emission are expected.

\section{Acknowledgements}

The support of the Namibian authorities and of the University of Namibia in facilitating the construction and operation of H.E.S.S. is gratefully acknowledged, as is the support by the German Ministry for Education and Research (BMBF), the Max Planck Society, the German Research Foundation (DFG), the French Ministry for Research, the CNRS-IN2P3, and the Astroparticle Interdisciplinary Programme of the CNRS, the U.K. Science and Technology Facilities Council (STFC), the IPNP of the Charles University, the Czech Science Foundation, the Polish Ministry of Science and Higher Education, the South African Department of Science and Technology and National Research Foundation, and by the University of Namibia. We appreciate the excellent work of the technical support staff in Berlin, Durham, Hamburg, Heidelberg, Palaiseau, Paris, Saclay, and in Namibia in the construction and operation of the equipment. Sabrina Casanova acknowledges the support from the Polish National Science Center under the Opus Grant UMO-2014/13/B/ST9/00945

\section{References}

[1] Hillas, A. M. 1995, Proc. of the 19th ICRC (La Jolla), 3, 445

[2] Ohm, S., van Eldik, C., \& Egberts, K. 2009, Astroparticle Physics, 31, 383

[3] de Naurois, M., \& Rolland, L. 2009, Astroparticle Physics, 32, 231

[4] Berge, D., Funk, S., \& Hinton, J. 2007, A\&A, 466, 1219

[5] Piron, F., Djannati-Atai, A., Punch, M., et al. 2001, A\&A, 374, 895

[6] Green, D. A. 2009, Bulletin of the Astronomical Society of India, 37, 45

[7] Abramowski, A., et al. 2014, ApJL, 794, L1

[8] Matsunaga, K., et al. 2001, PASJ, 53, 1003

[9] Mizuno, A., \& Fukui, Y. 2004, in Astronomical Society of the Pacific Conference Series, Vol. 317,59 
[10] de Wilt, P., et al. 2012, in American Institute of Physics Conference Series, Vol. 1505, American Institute of Physics Conference Series, 277-280

[11] Kothes, R., \& Dougherty, S. M. 2007, A\&A, 468, 993

[12] Lemoine-Goumard, M., et al. 2014, ApJL, 794, L16

[13] Aharonian, F. A., et al. 2007, A\&A, 464, 235

[14] Kelner, S. R., Aharonian, F. A., \& Bugayov, V. V. 2006, PhRvD, 74, 034018

[15] Abramowski, A., et al. 2014, MNRAS, 439, 2828

[16] Gotthelf, E. V., et al. 2014, ApJ, 788, 155 